ఠ

ORIGINAL RESEARCH

\title{
Sex differences in obesity and cognitive function in a cognitively normal aging Chinese Han population
}

\author{
This article was published in the following Dove Press journal: \\ Neuropsychiatric Disease and Treatment \\ 14 September 2017 \\ Number of times this article has been viewed
}

\section{Wei $\mathrm{Li}^{*}$ \\ Qi Qiu* \\ Lin Sun \\ Ling Yue \\ Tao Wang \\ Xia Li \\ Shifu Xiao \\ Alzheimer's Disease and Related Disorders Center, Shanghai Mental Health Center, Shanghai Jiao Tong University School of Medicine, Shanghai, People's Republic of China \\ *These authors contributed equally to this work}

Correspondence: Xia Li; Shifu Xiao Alzheimer's Disease and Related Disorders Center, Shanghai Mental Health Center, Shanghai Jiao Tong University School of Medicine, Wangping Road No 600, Xuhui District 200030, People's Republic of China

Tel +8621 64387250

Email ja_1023@hotmail.com; xiaoshifu@msn.com
Background: Sex differences in Alzheimer's disease and mild cognitive impairment have been well recognized. However, sex differences in cognitive function and obesity in cognitively normal aging Chinese Han population have not attracted much attention.

Objective: The aim of this study was to investigate the relationship between sex, obesity, and cognitive function in an elderly Chinese population with normal cognitive function.

Subjects and methods: A total of 228 cognitively normal aging participants (males/ females $=93 / 135$ ) entered this study. Their general demographic information (sex, age, and education) was collected by standardized questionnaire. Apolipoprotein E (APOE) genotype and serum lipid levels were measured. The Montreal Cognitive Assessment (MoCA) was used to assess participants' cognitive function.

Results: The prevalence of obesity in elderly women (18/133, 13.5\%) was significantly higher than that in men $(5 / 92,5.4 \%, P=0.009)$. Regression analyses showed that obesity was associated with drinking alcohol $(\mathrm{OR}=13.695, P=0.045)$ and triglyceride $(\mathrm{OR}=1.436, P=0.048)$ in women and limited to low-density lipoprotein $(\mathrm{OR}=11.829, P=0.023)$ in men. Women performed worse on the naming score for MoCA than men $(P<0.01)$. Stepwise linear regression analysis showed that education $(t=3.689, P<0.001)$ and smoking $(t=2.031, P=0.045)$ were related to the score of naming in female, while high-density lipoprotein $(t=-2.077, P=0.041)$ was related to the score of naming in male; however, no correlation was found between body mass index and cognitive function in both male and female $(P>0.05)$.

Conclusion: Our finding suggests that there are significant sex differences in obesity and specific cognitive domains in aging Chinese Han population with normal cognitive function.

Keywords: sex difference, obesity, cognitive function, lipids, elderly Chinese

\section{Introduction}

Sex differences in cognitive impairment in adulthood and aging have been well demonstrated. ${ }^{1}$ Accumulating epidemiological studies suggest that women are disproportionately affected by Alzheimer's disease (AD) in terms of both disease prevalence and severity. ${ }^{2}$ Compared to men with $\mathrm{AD}$, female $\mathrm{AD}$ patients tend to have a worse score on a variety of neuropsychological tasks, ${ }^{3}$ smaller hippocampal volumes, ${ }^{4}$ and more total brain atrophy and temporal lobe degeneration. ${ }^{5}$ However, sex differences in cognitive function in cognitively normal aging Chinese Han population have not attracted much attention. A population-based study shows that cognitive normal elderly men perform better than women in most cognitive abilities at baseline but have faster rates of decline in working memory, ${ }^{6}$ while other studies suggest that women have a better performance than men in tasks requiring cognitive flexibility such as the discrimination reversal learning. ${ }^{7}$ So the relevant research conclusions are contradictory. 
Obesity is a serious public health problem in China, and there is a significant difference between men and women in the prevalence of obesity and its influencing factors. ${ }^{8}$ Dozens of studies have shown that obesity is associated with decrements in cognitive function, independently of other cardiovascular risk factors. ${ }^{9}$ And, it is also proved to be a risk factor for developing dementia. ${ }^{10}$ Based on these previous studies, we hypothesize that there might also be some sex differences in the effects of obesity on cognitive function. For example, Hwang et $\mathrm{al}^{11}$ found that male mice are more vulnerable than the females to the impacts of high-fat diet on weight gains, metabolic alterations, deficits of learning, and hippocampal synaptic plasticity. Another study based on the African Americans points out that obesity tends to be more problematic for women's verbal memory performance, but for men, it is more problematic for their nonverbal memory performance. ${ }^{12}$ However, up to now, there have been no similar studies in China.

There is evidence that apolipoprotein metabolism changes with age, and it also has been implicated in cognitive decline and obesity in the elderly. ${ }^{13}$ The majority of apolipoproteins are constituents of lipoprotein particles, such as triglyceride (TG), cholesterol, very low-density lipoproteins, low-density lipoproteins (LDLs), and high-density lipoproteins (HDLs), which transport lipids between tissues for fuel and cholesterol metabolism. ${ }^{13}$ However, the relationships between apolipoproteins and the risk of cognitive impairment and obesity are not very consistent. For example, Reitz et $\mathrm{al}^{14}$ found that LDL is a risk factor for cognitive decline, while there is no association between HDL and cognitive impairment, but then they also prove that HDL can lower the risk of cognitive impairment. ${ }^{15}$ Despres ${ }^{16}$ thought that HDL is a main feature of the dyslipidemia associated with abdominal obesity, while Hokanson and Austin ${ }^{17}$ held up that high plasma TG concentration is the core factor for obesity. Numerous studies have shown that lipid metabolism is influenced by sex differences ${ }^{18}$ and Apolipoprotein E (APOE) genotypes. ${ }^{19}$ However, it is not well established how levels of these proteins differ with sex and whether the level of protein is related to healthy aging and cognition in later life. Therefore, the purpose of the present study is to address the sex differences in 1) cognitive function, 2) obesity, and 3) metabolic disturbances and their associated risk factors in aging Chinese Han population with normal cognitive function.

\section{Subjects and methods} Subjects

This was a cross-sectional investigation conducted in Shanghai from 2011 to $2012,{ }^{20}$ and 228 cognitively normal aging subjects were included in our study. They had undergone a screening process that included medical history, physical and neurological examinations, laboratory tests, and MRI scans. And, the following information (age, sex, years of education, history of smoking, and drinking alcohol) was obtained by a face-to-face interview. Beyond that, all the study subjects also needed to meet the following criteria: 1) Han Chinese, $>60$ years old; 2) denied memory or any other cognitive impairment; 3 ) without major medical abnormalities, including central nervous system diseases, unstable, acute or life-threatening medical illness (eg, cancer and infection); and 4) was able to complete the entire inspection. Individuals with a history of mild cognitive impairment, dementia, depression, and other disorders that might affect cognitive function were excluded. The Beijing version of Montreal Cognitive Assessment (MoCA) was used to assess their cognitive function. This screening test takes $\sim 10-15 \mathrm{~min}$ to administer and consists of 30 items measuring eight cognitive domains (such as visuospatial abilities, naming, attention, language function, fluency, abstract ability, delay recall, and orientation), and it is considered to be a brief and easy screening tool for accurately testing cognitive dysfunction. ${ }^{21}$ The assessment was performed by four psychometricians who had received concurrent training on MoCA.

Before this study was initiated, all the subjects had signed an informed consent and ethical approval was obtained from Shanghai Mental Health Center.

\section{Measurement of anthropometric variables, body mass index (BMI), fasting blood glucose, and lipid profile}

BMI was calculated as weight divided by height squared $\left(\mathrm{kg} / \mathrm{m}^{2}\right)$. In China, BMI $<18.5$ was defined as lean, while BMI $\geq 28$ was defined as obesity. ${ }^{22}$ Based on these criteria, our participants were classified as lean with BMI $<18.5$ and obese with BMI $\geq 28$.

Peripheral blood samples were collected between 7 and 9 am after an overnight fast. Ethylenediaminetetraacetic acid-containing tubes and clot activating gel-containing serum separator tubes were used for the isolation of DNA and biochemical parameters, respectively. Plasma glucose, TG, cholesterol, HDL, and LDL were measured by using Olympus AU2700 automatic biochemical analyzer (Beckman Coulter Inc., Carlsbad, CA, USA).

\section{Genotyping of APOE}

Genomic DNA was extracted from peripheral blood by using a Blood Genomic DNA Extraction Kit (Qiagen NV, Venlo, the Netherlands). Multiplex amplification refractory mutation 
system polymerase chain reaction was used to determine the APOE genotype. According to the method previously described, ${ }^{23}$ these subjects were divided into three groups, E2 (e2/e2 and e2/e3), E3 (e3/e3), and E4 (e2/e4, e3/e4, and $\mathrm{e} 4 / \mathrm{e} 4) .{ }^{24}$

\section{Data analysis}

Demographic and biochemical indexes and MoCA score were analyzed by using independent $t$-tests for the continuous variables and $\chi^{2}$ test for the categorical variables. Furthermore, a one-way analysis of covariance was used for group comparisons, after adjusting for demographic and biochemical indexes. Cross-comparisons were performed to calculate the prevalence of obesity by sex groups. Correlations between demographic and biochemical indexes were examined by Pearson's correlation coefficients. Finally, stepwise multiple regression analyses were used to examine the relationships between obesity, cognition, and other variables in male and female groups. Covariates in these stepwise forward entry models included those with significant differences shown in the univariate analyses. All statistical analyses used SPSS Version 17.0 with two-tailed $P$-values of 0.05 .

\section{Results \\ Sex differences in the general demographic data}

As shown in Table 1, the average years of education for male were higher than those of female $(P<0.05)$. And, the percentage of smokers and drinkers in men was significantly higher than in women $(P<0.001)$.

\section{Sex differences in APOE genotypes, $\mathrm{BMI}$, and biochemical indexes}

There was no statistical difference $(P>0.05)$ in the APOE genotypes between male and female. However, the prevalence of obesity (BMI $\geq 28$ ) in female $(9 / 135,6.67 \%)$ was three times higher than that in men $(2 / 93,2.15 \%)$. After controlling education, smoking, and drinking, the difference was still significant ( $\mathrm{OR}=0.374, P=0.044)$.

Higher cholesterol, HDL, and LDL plasma levels were found in female than in male $(P<0.05)$. However, the plasma level of fasting blood glucose in female was lower than that in male $(P<0.05)$, while there was no statistical difference $(P>0.05)$ in TG between two groups.

Correlation analysis suggested that the BMI of male was correlated with HDL ( $r=-0.261, P=0.013)$, while age, education, smoking, drinking, APOE genotypes, fasting blood glucose, TG, cholesterol, LDL, and the total score of MoCA were not correlated with BMI $(P>0.05)$.
Table I Sex difference in Chinese Han people with normal cognitive function

\begin{tabular}{|c|c|c|c|c|}
\hline & $\begin{array}{l}\text { Male } \\
(n=93)\end{array}$ & $\begin{array}{l}\text { Female } \\
(n=135)\end{array}$ & $F$ or $\chi^{2}$ & $P$-value \\
\hline Age (years) & $71.08 \pm 7.964$ & $70.26 \pm 8.152$ & 0.562 & 0.454 \\
\hline Education (years) & $10.74 \pm 3.756$ & $8.85 \pm 4.228$ & 11.919 & $0.00 I^{*}$ \\
\hline Smoking (yes/no) & $49 / 44$ & $3 / 132$ & 76.659 & $0.000^{*}$ \\
\hline Drinking (yes/no) & $38 / 55$ & $3 / 132$ & 56.469 & $0.000 *$ \\
\hline BMI $\left(\mathrm{kg} / \mathrm{m}^{2}\right)$ & & & 6.868 & $0.032 *$ \\
\hline Lean $(<\mid 8.5)$ & $2(2.2 \%)$ & $9(6.7 \%)$ & & \\
\hline Normal (I8.5-27.9) & 85 (91.4\%) & 106 (78.5\%) & & \\
\hline Obese $(\geq 28)$ & $5(5.4 \%)$ & $18(13.3 \%)$ & & \\
\hline APOE & & & 0.743 & 0.690 \\
\hline E2 (\%) & $13(14.0 \%)$ & $22(16.3 \%)$ & & \\
\hline E3 (\%) & 61 (65.6\%) & 91 (67.4\%) & & \\
\hline E4 (\%) & 19 (20.4\%) & $22(16.3 \%)$ & & \\
\hline \multicolumn{5}{|l|}{ Biochemical } \\
\hline $\begin{array}{l}\text { Fasting blood } \\
\text { glucose }(\mathrm{mg} / \mathrm{dL})\end{array}$ & $5.94 \pm 2.155$ & $5.26 \pm 1.266$ & 8.300 & $0.004 *$ \\
\hline Triglyceride (mg/dL) & $1.79 \pm 1.423$ & $1.90 \pm 1.324$ & 0.341 & 0.560 \\
\hline Cholesterol (mg/dL) & $4.43 \pm 0.926$ & $5.19 \pm 1.205$ & 24.165 & $0.000 *$ \\
\hline $\begin{array}{l}\text { High-density } \\
\text { lipoprotein }(\mathrm{mg} / \mathrm{dL})\end{array}$ & $1.10 \pm 0.245$ & $1.21 \pm 0.264$ & 10.365 & $0.00 I^{*}$ \\
\hline $\begin{array}{l}\text { Low-density } \\
\text { lipoprotein }(\mathrm{mg} / \mathrm{dL})\end{array}$ & $2.60 \pm 0.736$ & $3.11 \pm 1.012$ & 16.845 & $0.000 *$ \\
\hline \multicolumn{5}{|l|}{$\mathrm{MoCA}$} \\
\hline $\begin{array}{l}\text { Visual space } \\
\text { structure }\end{array}$ & $3.85 \pm 1.283$ & $3.45 \pm 1.417$ & 0.067 & 0.796 \\
\hline Naming & $2.64 \pm 0.675$ & $2.17 \pm 0.827$ & 13.006 & $0.000 *$ \\
\hline Attention & $5.60 \pm 0.849$ & $5.40 \pm 0.95 \mathrm{I}$ & 0.010 & 0.920 \\
\hline Language function & $\mathrm{I} .2 \mathrm{I} \pm 0.778$ & $1.21 \pm 0.826$ & 2.267 & 0.134 \\
\hline Fluency & $0.95 \pm 0.229$ & $0.93 \pm 0.250$ & 0.037 & 0.849 \\
\hline Abstract ability & $1.37 \pm 0.737$ & $1.09 \pm 0.787$ & 2.440 & 0.120 \\
\hline Delay recall & $2.61 \pm 1.619$ & $2.69 \pm 1.656$ & 2.066 & 0.152 \\
\hline Orientation & $5.89 \pm 0.479$ & $5.88 \pm 0.406$ & 1.301 & 0.255 \\
\hline MoCA total & $25.32 \pm 3.76 \mathrm{I}$ & $22.81 \pm 4.659$ & 3.646 & 0.058 \\
\hline
\end{tabular}

Notes: Data shown as mean \pm SD unless otherwise stated. $* p<0.05$ Abbreviations: APOE, Apolipoprotein E; BMI, body mass index; MoCA, Montreal Cognitive Assessment.

We also found that the BMI of female was correlated with cholesterol $(r=0.248, P=0.004)$ and LDL $(r=0.190, P=0.029)$. Next, by using the stepwise multiple regression analysis, we proved that cholesterol was significantly associated with BMI in female $(B=0.700, t=2.762, P=0.007)$.

Logistic regression analysis showed that obesity was significantly associated with $\mathrm{LDL}(\mathrm{OR}=11.829, P=0.023)$ in the male population, while drinking $(\mathrm{OR}=13.695, P=0.045)$ and TG $(\mathrm{OR}=1.436, P=0.048)$ were risk factors for obesity in female.

\section{Sex differences in cognitive function}

The covariance analysis (control of age and education) showed that the average score of male in naming was higher than that of female $(P<0.05)$. Figure 1 shows the difference in the MoCA naming scores between men and women. Stepwise linear regression analysis showed that HDL $(t=-2.077$, 


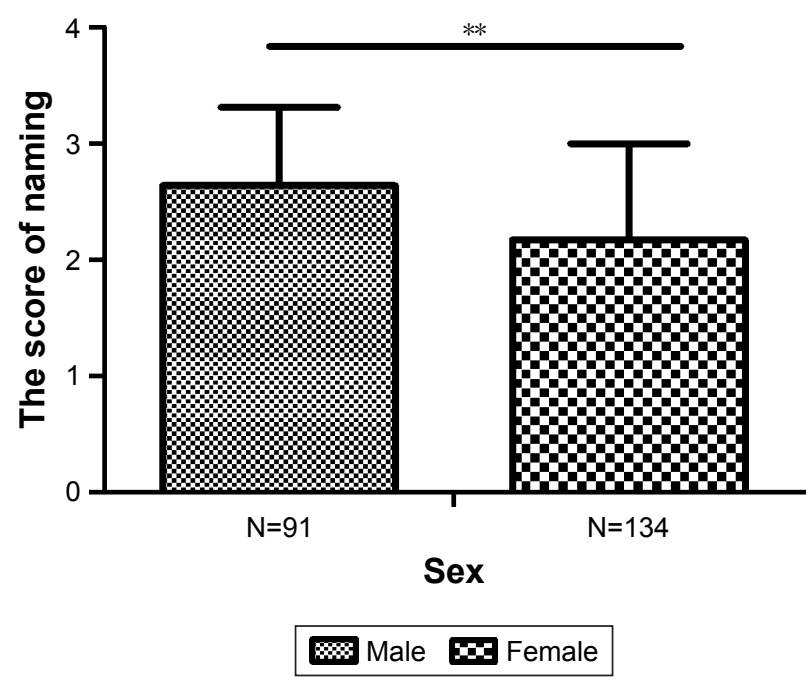

Figure I Sex difference in the score of naming. Note: ${ }^{* *} P<0.05$ male vs female.

$P=0.041)$ was related to the score of naming test in male; while education $(t=3.689, P<0.001)$ and smoking $(t=2.031$, $P=0.045$ ) were related to the score of naming in female. However, no correlation was found between BMI and cognitive function in both male and female $(P>0.05)$.

\section{Discussion}

To our best knowledge, this is the first study to explore the sex differences in cognitive function and obesity in aging Chinese Han population with normal cognitive function. Most studies support that males have an advantage on spatial tasks, while females perform better on verbal tasks. ${ }^{25}$ However, in our study, we found that men had better language functions, even when we had controlled for age, education, and other related factors. And, such inconsistencies might be attributable to the heterogeneity in study design, sex differences in the lipid profiles, and etiology of dementia as well as methods cognitive assessment. Previous studies ${ }^{26}$ were generally limited to global cognition, but in our study, we also compared the differences between men and women in specific areas of cognition. Although there was no statistical difference $(P>0.05)$ in MoCA total scores between men and women, we found that men had higher naming scores on MoCA than women. So we came to conclusion that although the language function of females is better than that of males, it is not obvious for the specific language task. ${ }^{25}$ By using stepwise linear regression analysis, we also found that $\operatorname{HDL}(t=-2.077, P=0.041)$ was related to the score of naming in male, while education ( $t=3.689, P<0.001)$ and smoking $(t=2.031, P=0.045)$ were related to the score of naming in female. Contrary to our finding, Reitz et al ${ }^{14}$ pointed out that higher levels of HDL-C were associated with a decreased risk of both probable and possible AD. Therefore, our conclusions were not very consistent and racial differences might have a bearing on the results.

The prevalence of obesity has been increasing in China, with more cases detected in the younger population. ${ }^{27}$ Obesity is related to several diseases such as stroke, diabetes, and high blood pressure. ${ }^{28} \mathrm{Up}$ to now, the association between obesity and cognition is still controversial. ${ }^{29,30}$ In our study, we found that elderly women had a higher BMI and prevalence of obesity than elderly men, which was consistent with a previous study. ${ }^{31}$ There was evidence that the increased obesity rates in women were significantly influenced by sex, as the result of physiological changes in hormonal status during and after menopause and sex, due to changes in individual and social behavior, such as modifications in socioeconomic status and adoption of unhealthy life. ${ }^{32,33}$ In our study, we proved that drinking and high levels of TG would increase the risk of obesity in females. However, previous studies suggested that low levels of HDL but not TG were the risk factors for obesity in women. Thus, our results are partially consistent. ${ }^{34}$ Moreover, we found that high levels of LDL were risk factors for obesity only in elderly men, which was consistent with previous research findings. ${ }^{35}$ Although many studies have confirmed that obesity is an important risk factor for cognitive decline, ${ }^{36-38}$ Deckers et al ${ }^{39}$ pointed out that the association between obesity and cognitive decline would be confounded by the effect of age on rate of decline. Similarly, we also did not find any link between obesity and cognitive function in both elderly men and women with normal cognitive function. Since this was only a cross-sectional study, these conclusions also need to be validated by a large sample of longitudinal studies.

There are several limitations in our study. First, this is only a cross-sectional study and cannot show direct causality of cognitive decline or obesity with serum lipid levels. Second, since our sample size is relatively small and these results are preliminary, further validation is needed. Third, serum lipid levels can be influenced by environmental factors, cultural status, and dietary habits; unfortunately, we failed to evaluate these factors. Fourth, the pathophysiological changes of AD may begin in midlife and studies conducted in the late life of participants may have a potential shortcoming that aging-related pathological changes may outweigh the risk related to lipids.

\section{Conclusion}

The present study proved that sex differences existed in specific cognitive domains and the rate of obesity in aging 
Chinese Han population with normal cognitive function. Compared to men, elderly women had a worse ability of naming and a higher BMI and rate of obesity, making them more likely to suffer from chronic diseases, including AD; drinking and high levels of TGs would increase the risk of obesity in women, while LDL was a risk factor for obesity in men. Since this research is only a cross-sectional study and the sample size is valid, we still need a large sample of longitudinal studies to investigate the effects of sex differences on lipid metabolism and cognitive function.

\section{Acknowledgments}

This work was supported by grants from the China Ministry of Science and Technology (2009BAI77B03) and National Natural Science Foundation of China (number 81671402).

\section{Disclosure}

The authors report no conflicts of interest in this work.

\section{References}

1. Li R, Singh M. Sex differences in cognitive impairment and Alzheimer's disease. Front Neuroendocrinol. 2014;35(3):385-403.

2. Koran MEI, Wagener M, Hohman TJ; Alzheimer's Neuroimaging Initiative. Sex differences in the association between AD biomarkers and cognitive decline. Brain Imaging Behav. 2017;11(1):205-213.

3. Henderson VW, Buckwalter JG. Cognitive deficits of men and women with Alzheimer's disease. Neurology. 1994;44(1):90-96.

4. Apostolova LG, Dinov ID, Dutton RA, et al. 3D comparison of hippocampal atrophy in amnestic mild cognitive impairment and Alzheimer's disease. Brain. 2006;129(11):2867-2873.

5. Hua X, Hibar DP, Lee S, et al; Alzheimer's Disease Neuroimaging Initiative. Sex and age differences in atrophic rates: an ADNI study with n=1,368 MRI scans. Neurobiol Aging. 2010;31(8):1463-1480.

6. Li LW, Ding D, Wu B, Dong X. Change of cognitive function in U.S. Chinese older adults: a population-based study. J Gerontol A Biol Sci Med Sci. 2017;1(72):5-10.

7. Miletto Petrazzini ME, Bisazza A, Agrillo C, Lucon-Xiccato T. Sex differences in discrimination reversal learning in the guppy. Anim Cogn. 2017;8(8):1117-1124.

8. Fang C, Liang Y. Social disparities in body mass index (BMI) trajectories among Chinese adults in 1991-2011. Int J Equity Health. 2017; 16(1):146-149.

9. Elias MF, Elias PK, Sullivan LM, Wolf PA, D'Agostino RB. Lower cognitive function in the presence of obesity and hypertension: the Framingham heart study. Int J Obes Relat Metab Disord. 2003;27(2): 260-268.

10. Stewart R, Masaki K, Xue QL, et al. A 32-year prospective study of change in body weight and incident dementia: the Honolulu-Asia aging study. Arch Neurol. 2005;62(1):55-60.

11. Hwang LL, Wang CH, Li TL, et al. Sex differences in high-fat dietinduced obesity, metabolic alterations and learning and synaptic plasticity deficits in mice. Obesity. 2010;18(3):463-469.

12. Wright RS, Cole AP, Ali MK, Skinner J, Whitfield KE, Mwendwa DT Examining the influence of measures of adiposity on cognitive function in middle age and older African Americans. Arch Clin Neuropsychol. 2016;31(1):23-28

13. Muenchhoff J, Song F, Poljak A, et al. Plasma apolipoproteins and physical and cognitive health in very old individuals. Neurobiol Aging. 2017;55(5):49-60.
14. Reitz C, Tang MX, Luchsinger J, Mayeux R. Relation of plasma lipids to Alzheimer disease and vascular dementia. Arch Neurol. 2004;61(5): 705-714.

15. Reitz C, Tang MX, Schupf N, Manly JJ, Mayeux R, Luchsinger JA Association of higher levels of high-density lipoprotein cholesterol in elderly individuals and lower risk of late-onset Alzheimer disease. Arch Neurol. 2010;67(12):1491-1497.

16. Despres JP. Dyslipidaemia and obesity. Baillieres Clin Endocrinol Metab. 1994;8(3):629-660.

17. Hokanson JE, Austin MA. Plasma triglyceride level is a risk factor for cardiovascular disease independent of high-density lipoprotein cholesterol level: a meta-analysis of population-based prospective studies. J Cardiovasc Risk. 1996;3(2):213-219.

18. Lew J, Sanghavi M, Ayers CR, et al. Sex-based differences in cardiometabolic biomarkers. Circulation. 2017;135(6):544-555.

19. Tao QQ, Chen Y, Liu ZJ, et al. Associations between apolipoprotein E genotypes and serum levels of glucose, cholesterol, and triglycerides in a cognitively normal aging Han Chinese population. Clin Interv Aging. 2014;9(1):1063-1067.

20. Xiao S, Li J, Tang M, et al. Methodology of China's national study on the evaluation, early recognition, and treatment of psychological problems in the elderly: the China Longitudinal Aging Study (CLAS). Shanghai Arch Psychiatry. 2013;25(2):91-98.

21. Nasreddine ZS, Phillips NA, Bedirian V, et al. The Montreal Cognitive Assessment, MoCA: a brief screening tool for mild cognitive impairment. J Am Geriatr Soc. 2005;53(4):695-699.

22. Ji CY; Working Group on Obesity in China. Report on childhood obesity in China (1) - body mass index reference for screening overweight and obesity in Chinese school-age children. Biomed Environ Sci. 2005; 18(6):390-400.

23. Donohoe GG, Salomaki A, Lehtimaki T, Pulkki K, Kairisto V. Rapid identification of apolipoprotein E genotypes by multiplex amplification refractory mutation system PCR and capillary gel electrophoresis. Clin Chem. 1999;45(1):143-146.

24. Anderson GD, Temkin NR, Dikmen SS, et al. Haptoglobin phenotype and apolipoprotein E polymorphism: relationship to posttraumatic seizures and neuropsychological functioning after traumatic brain injury. Epilepsy Behav. 2009;16(3):501-506.

25. Andreano JM, Cahill L. Sex influences on the neurobiology of learning and memory. Learn Mem. 2009;16(4):248-266.

26. Lu Y, An Y, Yu H, et al. Sex-specific nonlinear associations between serum lipids and different domains of cognitive function in middle to older age individuals. Metab Brain Dis. 2017;4(3):21-24.

27. Xi B, He D, Hu Y, Zhou D. Prevalence of metabolic syndrome and its influencing factors among the Chinese adults: the China Health and Nutrition Survey in 2009. Prev Med. 2013;57(6): $867-871$.

28. Kwast BE. Quality of care in reproductive health programmes: monitoring and evaluation of quality improvement. Midwifery. 1998; 14(4):199-206.

29. Elias MF, Goodell AL, Waldstein SR. Obesity, cognitive functioning and dementia: back to the future. J Alzheimers Dis. 2012;30(2): 113-125.

30. Whitmer RA, Gunderson EP, Barrett-Connor E, Quesenberry CP $\mathrm{Jr}$, Yaffe K. Obesity in middle age and future risk of dementia: a 27 year longitudinal population based study. BMJ. 2005;330(7504): 1360-1363.

31. Slagter SN, van Waateringe RP, van Beek AP, van der Klauw MM, Wolffenbuttel BHR, van Vliet-Ostaptchouk JV. Sex, BMI and age differences in metabolic syndrome: the Dutch Lifelines Cohort study. Endocr Connect. 2017;4(18):207-210.

32. Krieger N. Genders, sexes, and health: what are the connections - and why does it matter? Int J Epidemiol. 2003;32(4):652-657.

33. EUGenMed Cardiovascular Clinical Study Group, Regitz-Zagrosek V, Oertelt-Prigione S, et al. Gender in cardiovascular diseases: impact on clinical manifestations, management, and outcomes. Eur Heart J. 2016;37(1):24-34 
34. Kuk JL, Ardern CI. Age and sex differences in the clustering of metabolic syndrome factors: association with mortality risk. Diabetes Care. 2010;33(11):2457-2461.

35. Millan-Nunez J, Mantilla-Morato T, Toro R, Millan-Perez JJ, MangasRojas A; Scientific Committee of Hypertriglyceridemia Registry of Spanish Society of Arteriosclerosis (SEA). Cardiometabolic risk related to the association of hypertriglyceridemia-low HDLc. Curr Pharm Des. 2016;22(3):365-371.

36. McIntyre RS, Mansur RB, Lee Y, et al. Adverse effects of obesity on cognitive functions in individuals at ultra high risk for bipolar disorder: results from the global mood and brain science initiative. Bipolar Disord. 2017;19(2):128-134.
37. Rodriguez-Casado A, Toledano-Diaz A, Toledano A. Defective insulin signalling, mediated by inflammation, connects obesity to Alzheimer disease; relevant pharmacological therapies and preventive dietary interventions. Curr Alzheimer Res. 2017;3(16):167-171.

38. Beydoun MA, Beydoun HA, Wang Y. Obesity and central obesity as risk factors for incident dementia and its subtypes: a systematic review and meta-analysis. Obes Rev. 2008;9(3):204-218.

39. Deckers K, Van Boxtel MPJ, Verhey FRJ, Kohler S. Obesity and cognitive decline in adults: effect of methodological choices and confounding by age in a longitudinal study. J Nutr Health Aging. 2017;21(5): 546-553.

\section{Publish your work in this journal}

Neuropsychiatric Disease and Treatment is an international, peerreviewed journal of clinical therapeutics and pharmacology focusing on concise rapid reporting of clinical or pre-clinical studies on a range of neuropsychiatric and neurological disorders. This journal is indexed on PubMed Central, the 'PsycINFO' database and CAS, and is the official journal of The International Neuropsychiatric Association (INA). The manuscript management system is completely online and includes a very quick and fair peer-review system, which is all easy to use. Visit http://www.dovepress.com/testimonials.php to read real quotes from published authors.

Submit your manuscript here: http://www.dovepress.com/neuropsychiatric-disease-and-treatment-journal 